\title{
A Study on Bacterial Contamination of Multidose Vaccine Vials in Southeast of Iran
}

\author{
Seyed Mehdi Tabatabaei, ${ }^{1,}$ Alireza Salimi Khorashad, ${ }^{1}$ Sahar Shahraki, ${ }^{3}$ and Feiz Mohammad Elhami ${ }^{3}$ \\ ${ }^{1}$ Infectious Diseases and Tropical Medicine Research Centre, Zahedan University of Medical Sciences, Zahedan, IR Iran \\ ${ }^{2}$ Public Health Reference Laboratory, Zahedan University of Medical Sciences, Zahedan, IR Iran \\ ${ }^{3}$ Immunization Department, Sistan and Balouchistan Provincial Health Centre, Zahedan University of Medical Sciences, Zahedan, IR Iran \\ "Corresponding author: Seyed Mehdi Tabatabaei, Infectious Diseases and Tropical Medicine Research Centre, Zahedan University of Medical Sciences, Zahedan, IR Iran. Tel: \\ +98-5433438801, Fax: +98-5433438800, E-mail: zu.healthdeputy@gmail.com
}

Received 2017 March 04; Revised 2017 April 13; Accepted 2017 April 15.

\begin{abstract}
Background: Contaminated multipledose vaccine vials (MDVVs) have been implicated in the outbreaks of pyogenic abscesses and other bacterial infections. In this study, we aimed to investigate the prevalence of bacterial contamination of MDVVs.

Methods: Over 3 months, a total of 3640 in-use MDVVs were collected from 39 urban health centres and health posts in Zahedan, southeast of Iran and inoculated on appropriate bacterial growth media. The bacteria were identified using the methods described by the American type culture collection (ATCC).

Results: Bacterial contamination was identified in 6 out of 3640 (0.2\%)MDVVs. The isolated microorganisms included Staphylococcus epidermidis (3 vials), Staphylococcus saprophyticus (2 vials), and Escherichia coli (1 vial).

Conclusions: The present results revealed some breaches in safe injection practices and noncompliance with aseptic techniques in vaccination services provided by urban health centres and health posts. This article highlights the importance of basic infection control training and the need for increased awareness of safe injection practices among healthcare workers.
\end{abstract}

Keywords: Vaccination, Safe Injection, Multidose Vaccine Vial, Contamination

\section{Background}

A multidose vaccine vial (MDVV) typically contains an antimicrobial preservative to help prevent the growth of bacteria. However, these preservatives can prevent bacterial contaminations only if healthcare workers comply with safe injection practices (1). The World Health Organization (WHO) estimates show that out of 12 billion injections given annually, 5\% are administered for immunization (2). It is well-documented that at least $50 \%$ of injections are unsafe in developing countries (3). Unsafe injection can give rise to transmission of a wide variety of pathogens, including human immunodeficiency virus (HIV), hepatitis B virus (HBV), and hepatitis C virus (HCV) (4).

In 2000, WHO estimated that unsafe injection practices accounted for 32\% ( 21 million cases), 40\% (2 million cases) and 5\% (26 thousand cases) of the global burden of new HBV, HCV, and HIV infections, respectively (5). Unsafe injection practices are associated with more than 1.3 million deaths, and the cost of unsafe injections are estimated at approximately 535 million dollars per year (6). During 2000 - 2010, the absolute number of HIV and HCV infections, transmitted through unsafe injection practices, has shown a reduction of $87 \%$ and $83 \%$, respectively (7). How- ever, in some developing countries, unsafe injections are commonplace and still pose a major public health challenge.

Injection safety is an important component of immunization programs. According to WHO, a safe injection does not harm the recipient, does not expose the vaccinator to any avoidable risks, and does not produce dangerous waste for the surrounding community (8). Failure of vaccinators and healthcare workers to comply with aseptic techniques may give rise to bacterial contaminations of vaccine vials, which is likely to result in adverse consequences following immunization.

Outbreaks of pyogenic abscesses after diphtheria, tetanus toxoids, and pertussis (DTP) vaccination, resulting from vaccine vials contaminated with group A Streptococcus and Staphylococcus aureus, have been reported (9-11). Factors contributing to abscess formation following immunization include faulty technique, site, and route of inoculation, microbiologic contamination, and hypersensitivity (12).

To the best of our knowledge, no studies have been carried out on the prevalence of vaccine vial contamination in Iran. Only several studies have investigated bacterial contamination of multidose vials (MDVs) in hospital settings. Bacterial contamination was reported in $5.6 \%$ of MDVs, ad- 
ministered in a major referral teaching hospital in Shiraz, Southwest of Iran, and the most commonly identified organism was Staphylococcus epidermidis (13).

Similarly, microbial contamination was identified in $5.36 \%$ of single- and multidose vials in a pulmonary teaching hospital. The highest contamination rate was reported in vials, used in the interventional bronchoscopy unit, and Staphylococcus epidermidis was identified as the most frequently isolated pathogen (14). However, contamination rates of $1 \%$ or lower have been reported for multiple use vials in Iran, as well as some developed countries $(15,16)$.

\section{Objectives}

The present study was carried out to investigate the prevalence of bacterial contamination of MDVVs used in urban health centres and health posts of Zahedan, Southeast of Iran.

\section{Methods}

\subsection{Setting and Sample Size Calculation}

Based on the data from 2 Iranian studies $(13,14)$ indicating the prevalence of bacterial contamination of MDVs (approximately 5\%), the sample size was estimated at 3640 vials $(\alpha, 0.05 ; \beta, 0.2 ; \mathrm{d}, 0.01)$ while considering the effect of the study design. We included all urban health centres and health posts in Zahedan, Southeast of Iran. The number of vaccine vials, collected from each health centre or health post, was determined using probability proportional to size, based on the number of vaccine doses administered at the health centres or health posts over the last year.

\subsection{Collection of Vaccine Vial Samples}

Commonly used MDVVs, including bacille calmetteguerin (BCG), measles, mumps, and rubella (MMR), and DTP, were collected over 3 months between January and March 2015. The samples only included in-use vaccine vials, and we made sure that the expiration date of the vaccine had not passed. The date and time of sampling, vaccine vial type and manufacturer, batch number, opening time and date, vaccine storage condition (refrigerator or vaccine carrier), and expiration date were recorded. All the samples were moved to the public health reference laboratory of Zahedan University of Medical Sciences under cold chain conditions and were cultured upon arrival; otherwise the samples were stored at the refrigeration temperature of $4^{\circ} \mathrm{C}$. An experienced laboratory expert performed all the cultures.

\subsection{Isolation and Identification of Contaminants}

The vaccine vials were well shaken before sampling, and the vial gums were disinfected using 70\% ethyl alcohol. The vials were inverted and volume of $100 \mu \mathrm{L}$ was removed with a sterile needle and syringe. Vaccine samples were inoculated on blood and eosin methylene blue (EMB) agar medium and incubated under aerobic conditions at $37^{\circ} \mathrm{C}$ for 48 hours. All the isolates were subjected to colony morphology, culture studies, and Gram staining. Confirmatory identification was carried out, using the methods described by the American type culture collection (ATCC).

Categorical variables are presented as number and percentages. Data analysis was performed using SPSS version 20 (Chicago, IL, USA).

\section{Results}

A total of 3640 MDVVs were collected from 39 urban health centres and health posts. The majority of vaccine vials were MMR and DTP (Table 1). Only $2 \%$ of the samples were collected from vaccine carriers, and the rest of the vials were obtained from vaccination unit refrigerators. The opening date was not marked on 992 (27.3\%) of vaccine vials. Overall, 2318 (63.7\%) vaccine vials were locally produced, while the rest of the samples were imported. Bacterial contamination was identified in $6(0.2 \%)$ investigated vaccine vials.

The 6 contaminated vaccine vials included 3 MMR, 2 DTP and 1 Pentavalent vaccines (Table 2). The opening date was marked on all the contaminated vaccine vials and all but one were collected from refrigerator. The most frequently isolated microorganisms included Staphylococcus epidermidis (3 vials), Staphylococcus saprophyticus (2 vials), and E. coli (1 vial).

\section{Discussion}

The results of the present study showed a contamination rate of $0.2 \%$ in opened in-use MDVVs, collected from urban health centres and health posts. In the review of the available literature, we found no studies on the prevalence of bacterial contamination in human vaccine vials. Our findings, however, are in agreement with the estimated overall risk of extrinsic contamination (introduced into the system during use) of 0.5 per 1000 MDVs of other medications (17).

In the present study, the contamination rate in MDVVs was much lower than the rates reported for MDVs in hospital settings of Iran (approximately 5\%) $(13,14)$. MDVs used in hospital settings are normally kept in the immediate patient treatment area, and inadvertent contamination of 
Table 1. Characteristics of Sampled Multi-Dose Vaccine Vials $(\mathrm{N}=3640)$

\begin{tabular}{|c|c|c|}
\hline Variable & Frequency & Percent \\
\hline \multicolumn{3}{|l|}{ Vaccine type } \\
\hline BCGa & 64 & 1.8 \\
\hline DTP b & 906 & 24.9 \\
\hline $\mathrm{DT}^{\mathrm{a}}$ & 16 & 0.4 \\
\hline $\mathrm{Td}^{\mathrm{b}}$ & 121 & 3.3 \\
\hline Hepatitis B & 400 & 11.0 \\
\hline MMRe & 1752 & 48.1 \\
\hline Pentavalent ${ }^{\mathrm{c}}$ & 381 & 10.5 \\
\hline \multicolumn{3}{|l|}{ Vaccine storage } \\
\hline Fridge & 3568 & 98.0 \\
\hline Vaccine Carrier & 72 & 2.0 \\
\hline \multicolumn{3}{|l|}{ Marking open date } \\
\hline No & 992 & 27.3 \\
\hline Yes & 2648 & 72.7 \\
\hline \multicolumn{3}{|l|}{ Vaccine source } \\
\hline Imported & 1322 & 36.3 \\
\hline Locally produced & 2318 & 63.7 \\
\hline \multicolumn{3}{|l|}{ Bacterial contamination } \\
\hline No & 3634 & 99.8 \\
\hline Yes & 6 & 0.2 \\
\hline
\end{tabular}

Abbreviations: BCG, Bacille Calmette-Guerin; DTP, Diphtheria, Tetanus, Pertussis; MMR, Measles, Mumps, Rubella.

${ }^{a}$ DT: Pediatric diphtheria and tetanus vaccine.

${ }^{\mathrm{b}} \mathrm{Td}$ : Older than 7 years children and adults tetanus and diphtheria vaccine.

${ }^{\mathrm{c}}$ Pentavalent: DTP-Hepatitis B-Hemophilus influenza type B.

the vial is more likely to happen through direct or indirect contact with potentially contaminated surfaces or equipments (14).

It has been well documented that poor aseptic techniques employed during successive applications of MDVs may result in serious and life-threatening infections in hospital settings (18). In comparison with hospital wards, it can be assumed that vaccination units are relatively cleaner and presumably less contaminated environments. This may partially explain the lower contamination rates of MDVVs in the present study, compared with figures related to MDVs used in hospital settings.

In addition to antigens, vaccines contain a variety of other ingredients, such as stabilizers, adjuvants, antibiotics, and preservatives (19). Preservatives, such as thimerosal, formaldehyde, or phenol derivatives, are added to vaccine vials to prevent bacterial or fungal growth in the event of accidental contamination, as might occur with repeated puncture of MDVs (19). Following fatal infections in vaccine recipients, in whom MDVVs without preservatives become contaminated during use, the manufacturers are legally required to ensure safety of vaccines by adding a preservative to MDVs (20).

However, preservatives, such as thimerosal, are likely to interfere with the efficacy of live-attenuated vaccines, such as MMR and BCG, which are exceptions to this rule. Since these vaccines do not contain preservatives, they should be discarded at the end of the immunization session (21). When formulated in multidose vials, preservatives are added to prevent bacterial contamination for other vaccines, such as diphtheria, DTP, diphtheria and tetanus toxoids (DT), tetanus toxoid (TT), hepatitis B, Hemophilus influenza type b (Hib), and influenza (21).

In our study, we detected bacterial contaminations in vaccine vials containing preservatives (i.e., DTP), as well as preservative-free vials (i.e., MMR). This finding underscores the importance of safe vaccine injection practices, regardless of the preservative content of vaccine vials. Outbreaks of pyogenic abscesses, related to unsafe injection practices, indicate that some vaccinators do not adhere to basic principles of infection control. Therefore, presence of preservatives in itself cannot guarantee contamination-free vaccination practices unless healthcare providers develop adequate skills to comply with the standards for sterile injection techniques.

Almost all vaccine vials currently used in the Iranian expanded program on immunization (EPI) include multidose formats. When using MDVVs, improper immunization practices could result in microbial contamination, leading to adverse events following immunization. Number of withdrawals from the vial, sterility of techniques employed by healthcare workers, injection of environmental air into the vial during extraction, duration of use, storage conditions, and presence of preservatives in the vial are among factors, which might affect the sterility of the vial content (15).

Single-dose vaccine vials may offer improved vaccine safety and significantly reduce the vaccine contamination rate. However, multidose formats, in general, are more cost-effective and show less cold-chain capacity (22). According to WHO multidose vial policy (MDVP, 2014), all opened MDVVs should be discarded within 6 hours of opening or at the end of the immunization session (23). Therefore, higher wastage rates and safety concerns, especially in terms of vaccine vial contamination, are major limitations for immunization services in developing countries.

With the improved vaccination coverage and global increase in the number of injections for vaccination, investment in vaccination injection safety measures, such as introduction of single-dose vaccine vials and use of autodis- 
Table 2. Characteristics of Contaminated Vaccine Vials

\begin{tabular}{|c|c|c|c|c|c|}
\hline Vaccine Type & Manufacturing Country & Volume (Doses) & Marking Opening Date & Storage & Organisms \\
\hline DTP & India & 10 & Yes & Fridge & Staph saprophyticus \\
\hline DTP & India & 5 & Yes & Fridge & Staph epidermidis \\
\hline MMR & Iran & 5 & Yes & Fridge & Staph epidermidis \\
\hline MMR & Iran & 5 & Yes & Fridge & Staph saprophyticus \\
\hline Pentavalent ${ }^{\mathrm{a}}$ & India & 10 & Yes & Fridge & Staph epidermidis \\
\hline MMR & Iran & 5 & Yes & Vaccine carrier & E.Coli \\
\hline
\end{tabular}

Abbreviations: DTP, Diphtheria, Tetanus, Pertussis; Staph, Staphylococcus; MMR, Measles, Mumps, Rubella.

${ }^{\text {a }}$ Pentavalent: DTP-Hepatitis B-Hemophilus influenza type B.

able (AD) syringes, should be considered by public health authorities (6). Single-dose formats are the most appropriate for more expensive vaccines considering the potential economic impact of various single-dose versus multidose vaccine vials in terms of costs related to production, medical waste disposal, and storage (22).

The mean daily patient arrival thresholds for each vaccine format have been proposed as a major determinant for choosing between single-dose and multi-dose vaccine vials, with a lower patient demand in favour of fewer dose formats (24). According to the results of a study on an economic computational model, the estimated thresholds for choosing fewer dose formats for multidose measles (MEA), hemophilusinfluenzae type B (Hib), BCG, yellow fever (YF), and pentavalent (DTP-hepatitis B-hemophilus influenza type b) vaccines were $2,6,5,33$, and 5 patients/day, respectively (24).

In order to improve immunization coverage in the area that this study was conducted, supplementary immunization campaigns for different vaccines including MMR are being carried out with varied frequencies. Supplementary immunization activities have been considered as an opportunity to promote safe injection practices. In African countries, for instance, unsafe vaccination injections contributed to 22 million infections that accounted for $39 \%$ of all unsafe injections (25). As part of measles supplemental immunization activities (SIAs), replacing disposable and sterilizable syringes with $\mathrm{AD}$ syringes along with healthcare workers training was undertaken to improve injection safety in 19 (49\%) of 39 African countries, funded by global alliance for vaccines and immunization (GAVI) (25). There is some evidence that supports the effectiveness and sustainability of such interventions as almost all countries that received assistance to improve immunization injection safety continued to comply with standards of injection safety in their immunization programs following the completion of the project (26). Similarly, the government of Andhra Pradesh, India, in partnership with the program for appropriate technology in health (PATH), which is an international NGO, successfully implemented safe injection in immunization programs (27). As part of this project, a 'bundling' concept in the immunization safety was launched that included high quality training to staff along with the introduction of AD syringes and safety boxes. As another example, in a large scale rubella campaign in Bolivia, great emphasis was put on the issues of safe injection practices and the campaign served as the launching pad to promote safe vaccination practices both in the routine immunization program and in subsequent campaigns across Bolivia (28). In order to ensure vaccine safety and to limit human errors in vaccination practices, WHO has also launched the safe injection global network (SIGN) and in this project training of healthcare workers providing vaccination services has been at the centre of this project (29).

One of the strengths of the present study was a relatively large sample size that was collected from all urban health centres and health posts in Zahedan, southeast of Iran. However, one of the limitation of our study was that all the samples were obtained from urban health centres and health posts. Almost half of the population residing in this province are living in the rural areas and they receive primary health care services including vaccination through rural health centres and health houses. Given the fact that rural areas are more likely to be less developed in terms of basic health infrastructures and they are sometimes facing shortage of human resources, it could be assumed that breaches in safe injection practices during vaccination are more frequent in rural areas. Therefore, caution should be taken when extrapolating the results of this study to the rural areas.

In conclusion, this study showed the importance of adherence to infection control practices, in particular, following appropriate sterile precautions during immuniza- 
tion. We found that the contamination rate for MDVVs was very low, but still it is of concern in providing safe immunization services. Public health managers should communicate the potential dangers of improperly administered vaccine injections and to advocate for clean immunization practices. In order to ensure injection safety in immunization programs, along with providing proper equipments, such as the use of $\mathrm{AD}$ syringes and safety boxes, it is necessary to pay attention to sterile techniques, especially when withdrawing vaccine from MDVVs. Providing different presentations of the same vaccine, such as multi-dose vials in a high-volume public health setting and prefilled AD syringes for extended outreach, could also be of help.

\section{Acknowledgments}

This study was funded by Zahedan University of Medical Sciences. The authors are grateful to the healthcare staff working in the urban health centres and health posts who helped us with collecting data and sampling vaccine vials during the study period.

\section{Footnotes}

Authors' Contribution: Seyed Mehdi Tabatabaei planned the study design and coordinated the conduct of the study. He also carried out statistical analysis and interpretation of the data and drafted this paper. Alireza Salimi Khorashad supervised all the data collection process and participated in drafting the manuscript. Sahar Shahraki performed all the lab tests. Sahar Shahraki and Feiz Mohammad Elhami undertook the data collection during the study period. Authors approved of the final draft of the paper.

Financial Disclosure: The authors declared no financial support and disclosure.

Funding/Support: This research was funded by Zahedan University of Medical Sciences, I.R. of Iran.

\section{References}

1. Centers for Disease Control and Prevention . Injection Safety Centers for Disease Control and Prevention; 2015. [updated 2015]. Available from: http://www.cdc.gov/injectionsafety/.

2. Kermode M. Unsafe injections in low-income country health settings: need for injection safety promotion to prevent the spread of blood-borne viruses. Health Promot Int. 2004;19(1):95-103. [PubMed: 14976177].

3. Simonsen L, Kane A, Lloyd J, Zaffran M, Kane M. Unsafe injections in the developing world and transmission of bloodborne pathogens: a review. Bull World Health Organ. 1999;77(10):789-800. [PubMed: 10593026].

4. World Health Organization . Best practices for injections and related procedures toolkit World Health Organization; 2010. [updated 2010]. Available from: http://www.who.int/injection_safety/ 9789241599252/en/.
5. Hutin YJ, Hauri AM, Armstrong GL. Use of injections in healthcare settings worldwide, 2000: literature review and regional estimates. BMJ. 2003;327(7423):1075. doi: 10.1136/bmj.327.7423.1075. [PubMed: 14604927].

6. Miller MA, Pisani E. The cost of unsafe injections. Bull World Health Organ. 1999;77(10):808-11. [PubMed: 10593028].

7. Pepin J, Abou Chakra CN, Pepin E, Nault V, Valiquette L. Evolution of the global burden of viral infections from unsafe medical injections, 2000-2010. PLoS One. 2014;9(6):e99677. doi: 10.1371/journal.pone.0099677. [PubMed: 24911341].

8. World Health Organization . Injection safety: The SIGN alliance World Health Organization; 2016. [updated 2016]. Available from: http:// www.who.int/injection_safety/sign/en/.

9. Stetler HC, Garbe PL, Dwyer DM, Facklam RR, Orenstein WA, West $\mathrm{GR}$, et al. Outbreaks of group A streptococcal abscesses following diphtheria-tetanus toxoid-pertussis vaccination. Pediatrics. 1985;75(2):299-303. [PubMed: 3881728].

10. Simon PA, Chen RT, Elliott JA, Schwartz B. Outbreak of pyogenic abscesses after diphtheria and tetanus toxoids and pertussis vaccination. Pediatr Infect Dis J. 1993;12(5):368-71. doi: 10.1097/00006454199305000-00003. [PubMed: 8327295].

11. Greaves WL, Hinman AR, Facklam RR, Allman KC, Barrett CL, Stetler HC. Streptococcal abscesses following diphtheria-tetanus toxoidpertussis vaccination. Pediatr Infect Dis. 1982;1(6):388-90. doi: 10.1097/00006454-198211000-00005. [PubMed: 7163027].

12. Bernier RH, Frank JJ, Nolan TJ. Abscesses complicating DTP vaccination. Am J Dis Child. 1981;135(9):826-8. doi: 10.1001/archpedi.1981.02130330036011. [PubMed: 7282659].

13. Motamedifar M, Askarian M. The prevalence of multidose vial contamination by aerobic bacteria in a major teaching hospital, Shiraz, Iran, 2006. Am J Infect Control. 2009;37(9):773-7. doi: 10.1016/j.ajic.2008.12.009. [PubMed: 19362388].

14. Baniasadi S, Dorudinia A, Mobarhan M, Karimi Gamishan M, Fahimi F. Microbial contamination of single- and multiple-dose vials after opening in a pulmonary teaching hospital. Braz J Infect Dis. 2013;17(1):69-73. doi:10.1016/j.bjid.2012.09.005. [PubMed: 23294643].

15. Khalili H, Sheikhbabayi M, Samadi N, Jamalifar H, Dalili D, Samadi N. Bacterial contamination of single- and multiple-dose vials after multiple use and intravenous admixtures in three different hospitals in iran. Iran J Pharm Res. 2013;12(1):205-9. [PubMed: 24250590].

16. Mattner F, Gastmeier P. Bacterial contamination of multiple-dose vials: a prevalence study. Am J Infect Control. 2004;32(1):12-6. doi: 10.1016/j.ajic.2003.06.004. [PubMed:14755229].

17. Longfield R, Longfield J, Smith LP, Hyams KC, Strohmer ME. Multidose medication vial sterility: an in-use study and a review of the literature. Infect Control. 1984;5(4):165-9. doi: 10.1017/S0195941700059154. [PubMed: 6562087].

18. Manchikanti L, Falco FJ, Benyamin RM, Caraway DL, Helm Ii S, Wargo $\mathrm{BW}$, et al. Assessment of infection control practices for interventional techniques: a best evidence synthesis of safe injection practices and use of single-dose medication vials. Pain Physician. 2012;15(5):E573614. [PubMed: 22996856].

19. World Health Organization . Components of a vaccine World Health Organization; 2016. [updated 2016]. Available from: http://vaccinesafety-training.org/vaccine-components.html.

20. U.S. Food and Drug Administration. Thimerosal in vaccines questions and answers. U.S. Food and Drug Administration; 2015. [updated 2015]. Available from: http://www.fda.gov/BiologicsBloodVaccines/ Vaccines/QuestionsaboutVaccines/UCM070430.

21. World Health Organization . Thiomersal and vaccines: questions and answers World Health Organization; 2006. [updated 2006]. Available from: http://www.who.int/vaccine_safety/committee/topics/ thiomersal/questions/en/. 
22. Drain PK, Nelson CM, Lloyd JS. Single-dose versus multi-dose vaccine vials for immunization programmes in developing countries. Bull World Health Organ. 2003;81(10):726-31. [PubMed: 14758432]

23. World Health Organization . WHO policy statement: multi-dose vial policy (MDVP): handling of multi-dose vaccine vials after opening. The policy brief revises and replaces WHO Policy Statement: The use of opened multi-dose vials of vaccine in subsequent immunization sessions World Health Organization; 2014. [updated 2014]. Available from: http://apps.who.int/iris/handle/10665/135972.

24. Lee BY, Norman BA, Assi TM, Chen SI, Bailey RR, Rajgopal J, et al. Single versus multi-dose vaccine vials: an economic computational model. Vaccine. 2010;28(32):5292-300. doi: 10.1016/j.vaccine.2010.05.048. [PubMed: 20566395].

25. Hoekstra EJ, van den Ent MM, Dao H, Khalaf H, Salovaara A. Measles supplementary immunization activities and GAVI funds as catalysts for improving injection safety in Africa. J Infect Dis. 2011;204 Suppl 1:S190-7. doi: 10.1093/infdis/jir073. [PubMed: 21666161].
26. Levin A, Fang A, Hansen PM, Pyle D, Dia O, Schwalbe N. A global health partnership's use of time-limited support to catalyze health practice change: the case of GAVI's Injection Safety Support. PLoS One. 2010;5(9):e12986. doi: 10.1371/journal.pone.0012986. [PubMed: 20885995].

27. Kaipilyawar SB, Rao RG. Injection safety for immunisation-Andhra Pradesh experience. J Indian Med Assoc. 2005;103(4):222-5. [PubMed: 16173430].

28. Halkyer P, Azurduy R, Fuentes M, Van Dick AM, Ronveaux O. Putting safety first: ensuring safe vaccination practices during the 2006 rubella campaign in Bolivia. J Infect Dis. 2011;204 Suppl 2:S718-21. doi: 10.1093/infdis/jir443. [PubMed: 21954272].

29. Jodar L, Duclos P, Milstien JB, Griffiths E, Aguado MT, Clements CJ. Ensuring vaccine safety in immunization programmes-a WHO perspective. Vaccine. 2001;19(13-14):1594-605. doi: 10.1016/S0264410X(00)00358-3. [PubMed: 11166881]. 